\section{ORIGINAL} RESEARCH

Y. Loh

D. Kim

Z.-S. Shi

S. Tateshima

P.M. Vespa

N.R. Gonzalez

S. Starkman

J.L. Saver

R. Jahan

D.S. Liebeskind

G.R. Duckwiler

F. Viñuela

\title{
Higher Rates of Mortality but Not Morbidity Follow Intracranial Mechanical Thrombectomy in the Elderly
}

\author{
BACKGROUND AND PURPOSE: Mechanical thrombectomy is a promising means of recanalizing acute \\ cerebrovascular occlusions in certain situations. We sought to determine if increasing age adversely \\ affects prognosis.
}

\begin{abstract}
MATERIALS AND METHODS: We reviewed all Merci thrombectomy cases and compared patients younger than 80 years of age with older individuals. We compared these 2 age groups with respect to recanalization rates, hospital LOS, hemorrhagic transformation, and death and disability on discharge.
\end{abstract}

RESULTS: Elderly patients were more likely to die from their stroke than those younger than 80 years of age, regardless of recanalization success (48\% versus 15\%; OR, 5.5; 95\% Cl, 2.1-14.1). Among survivors, there was no difference in the probability of having a good functional outcome (mRS, $\leq 2)$ by discharge (38\% versus 40\%; OR, 0.9; 95\% Cl, 0.3-2.8). Hemorrhagic transformation did not vary between age groups.

CONCLUSIONS: Among patients undergoing mechanical thrombectomy for acute cerebrovascular occlusions, increased age conveys a higher rate of stroke-related death, but disability at discharge in this group is similar to that of younger survivors.

\begin{abstract}
ABBREVIATIONS: $\mathrm{AOL}=$ arterial occlusive lesion; $\mathrm{Cl}=$ confidence interval; $\mathrm{HT}=$ hemorrhagic transformation; IA = intra-arterial; ICA = internal carotid artery; LOS = length of stay; MCA = middle cerebral artery; $\mathrm{MERCI}=$ Mechanical Embolus Removal in Cerebral Ischemia; $\mathrm{mRS}=$ modified Rankin Scale; NIHSS = National Institutes of Health Stroke Scale; OR = odds ratio; PH = parenchymal hematoma; PTA = percutaneous transluminal angioplasty; rtPA = recombinant tissue plasminogen activator; $\mathrm{TIA}=$ transient ischemic attack
\end{abstract}

lons ntracranial mechanical thrombectomy is a promising ischemic stroke treatment. Since the introduction and US Food and Drug Administration clearance of the Merci retriever system (Concentric Medical, Mountain View, California) for recanalization of large-vessel thomboembolic occlusions, its use is ever-increasing. ${ }^{1,2}$ There is ongoing interest in the selection

Received October 29, 2009; accepted after revision January 9, 2010

From the Divisions of Neurosurgery (Y.L., P.M.V., N.R.G.) and Interventional Neuroradiology (Y.L., Z.-S.S., S.T., N.R.G., R.J., G.R.D., F.V.), and Department of Neurology (D.K., S.S., J.L.S., D.S.L,), David Geffen School of Medicine at the University of California, Los Angeles, Los Angeles, California; Neurovascular Service (Y.L.), Department of Medicine, Madigan Army Medical Center, Tacoma, Washington; and Department of Neurosurgery (Z.-S.S.), First Hospital of Sun Yet-sen University, Guangzhou, Guangdong, China.

The views expressed in this article are those of the authors and do not reflect the official policy or position of the Department of the Army, the Department of Defense, or the US Government.

All authors are or have been employees of the University of California, which holds several patents on retriever devices for stroke. Dr Duckwiler is a Scientific Advisor for and shareholder in Concentric Medical. Dr Liebeskind is a consultant for Concentric Medical. Dr Starkman has received grant funding for clinical trials from Concentric Medical and Genentech. Dr Saver is a scientific consultant for CoAxia, Concentric Medical, Talecris, Ferrer, AGA Medical, BrainsGate, PhotoThera, and Cygnis; has received lecture honoraria from Ferrer and Boehringer Ingelheim; has received support for clinical trials from Concentric Medical; and is a site investigator in multicenter trials sponsored by AGA Medical and the National Institutes of Health for which the University of California Regents received payments based on the number of subjects enrolled.

Please address correspondence to Yince Loh, MD, Neurovascular Service, Department of Medicine, Madigan Army Medical Center, Bldg 9040, Fitzsimmons Dr, Tacoma, WA 98431; e-mail: yincer@yahoo.com

Indicates open access to non-subscribers at www.ajnr.org

DOI 10.3174/ajnr.A2079 of appropriate candidates because it remains unclear which patients benefit the most.

The elderly population is predicted to double in a half century, and it is well established that older persons have a higher risk of stroke. ${ }^{3,4}$ Advanced age also carries with it the burden of decreased neuronal plasticity and an ever-decreasing pool of healthy neurons. A large intracranial vessel occlusion may irreversibly injure a higher proportion of neurons in an elderly patient than it would in a younger patient under similar circumstances, substantially impeding recovery of neurologic function. ${ }^{5}$ Thus, elderly patients may incur more disability regardless of treatment technique. ${ }^{6}$

The safety and effectiveness of acute stroke therapies, including intravenous and IA thrombolysis, have been studied in the older population. ${ }^{7,8}$ However, the efficacy of intracranial mechanical thrombectomy in this population is poorly explored. The purpose of this study was to examine, with respect to increasing age, our single-center experience with intracranial mechanical thrombectomy by using the Merci retriever.

\section{Materials and Methods}

\section{Patients and Techniques}

All consecutive patients undergoing Merci clot retrieval for large arterial occlusions from August 2002 to December 2007 were prospectively entered into the data base of our institution according to protocol approved by our local institutional review board. We retrospectively reviewed all anterior circulation occlusions involving the intracranial ICA and/or the M1 segment of the MCA. All posterior 
circulation occlusions were included. Tandem lesions were included if at least 1 of the occlusions involved any of the mentioned vessels.

In all cases, transfemoral cerebral angiography was performed to document arterial occlusion. Next, the diagnostic catheter was exchanged for an $8 \mathrm{~F}$ or $9 \mathrm{~F}$ balloon guiding catheter, or the guiding catheter was advanced coaxially over it. After angiographic visualization of the cervical internal carotid or vertebral origin, the artery was catheterized, if the procedure was safe. Once the guide catheter was in a stable position, it was attached and connected to continuous heparinized saline flush. A microcatheter and microwire was then advanced coaxially up to the occlusion. The microcatheter was positioned distal to the thrombus, and contrast was injected to confirm positioning and vessel diameter. Typically, the proximal balloon catheter tip was then inflated to arrest antegrade flow. The Merci retriever was then advanced into the microcatheter, and all loops were deployed distal to the thrombus. Next, a slow pull was applied to the entire microsystem under fluoroscopy. Gentle aspiration was made through the lumen of the guiding catheter during active extraction. Once the retriever began moving freely, blood was vigorously aspirated from the guiding catheter. The balloon catheter tip was deflated, and control angiography was performed. The procedure was concluded if satisfactory recanalization was achieved. If occlusion persisted, this procedure was repeated and was aborted if the surgeon thought that the clot could not be extracted or a maximum of 6 attempts was made. The angiographic result was graded according to the previously described AOL scale. ${ }^{9}$

We separated patients into 2 groups: those 80 years of age or older, and those younger. We compared the 2 groups with regard to demographic data, the presence of premorbid conditions, and aspects of the intervention. We also noted concomitant use of an IA thrombolytic or preintervention administration of an intravenous thrombolytic.

Patients underwent pre-Merci MR imaging to include diffusionweighted, fluid-attenuated inversion recovery, and gradient recalledecho sequences; intracranial time-of-flight MR angiography; and perfusion-weighted imaging. In patients unable to undergo MR imaging, CT including CT angiography and perfusion sequences was performed. Patients were typically selected for thrombectomy if the infarct volume was subjectively small and there was salvageable penumbra. All patients underwent noncontrast head CT immediately after the procedure. Multimodal MR or CT imaging similar to that in the pre-Merci study was performed from mostly 5 but up to 24 hours after thrombectomy.

In our primary analysis, we compared clinical outcome measures including preintervention and discharge NIHSS scores, hospital LOS, change in NIHSS score by discharge, discharge mRS score, and rate of stroke-related death. For these NIHSS comparisons, patients who died from their stroke were assigned the maximum NIHSS score.

As a secondary analysis, we compared the discharge NIHSS score, change in NIHSS score by discharge, and the probability of a good outcome ( $\mathrm{mRS}$ score, $\leq 2$ ) in survivors. We also examined the proportions of all types of HT according to the European Cooperative Acute Stroke Study definition. ${ }^{10}$ We then assessed rates of PH (defined as both types of PHs, $\mathrm{PH} 1$ and $\mathrm{PH} 2$ ). We also compared the distribution of symptomatic HT, defined as a decrease in NIHSS score by $\geq 4$ points or prompting surgical intervention.

We also conducted several post hoc analyses based on the results of our primary comparison. We first sought to determine if the cardioembolic stroke source had an independent impact on clinical outcome and stroke-related death. As another post hoc analysis, we selected a more homogenously aged subgroup from the younger

$\begin{aligned} & \text { Table 1: Cohort age distribution by deciles in patients undergoing } \\
& \text { Merci thrombectomy for acute ischemic stroke }\end{aligned}$
\begin{tabular}{lccc}
\hline Decile & Age (yr) & Decile & Age (yr) \\
\hline 1st & $14-39$ & 6 th & $71-74$ \\
2nd & $39-47$ & 7 th & $74-78$ \\
3rd & $48-56$ & 8 th & $78-83$ \\
4th & $56-64$ & 9 th & $84-86$ \\
5th & $65-70$ & 10th & $86-95$ \\
\hline
\end{tabular}

population (65-79 years of age) to compare with the octogenarian and older cohort to exclude stroke in the younger population. This was aimed at approximating a more typical acute ischemic stroke patient population. We compared this subgroup with the group 80 years of age and older with regard to clinical outcome measures.

Statistical analyses for categoric variables included the $\chi^{2}$ and Fisher exact tests when cell sizes were small and ORs for selected dichotomous comparisons. We examined continuous variables by using analysis of variance, and applied the Mann-Whitney $U$ test to measures with uneven distributions.

\section{Results}

A total of 106 patients met inclusion criteria. Among these patients, 75 were younger than of 80 years of age (median, 59 years; interquartile range, $46.5-71.5$ years), while 31 were 80 years of age or older (median, 85 years; interquartile range, 83-89). Forty-five percent were men, and the median pretreatment NIHSS score was 19 . Because of premorbid disability, 2 patients were excluded solely from the $\mathrm{mRS}$ analysis. One patient had a prolonged hospital stay from a nonstroke-related condition diagnosed during hospitalization and was excluded solely from the hospital LOS comparison. Eight patients younger than 80 years of age and 2 elderly patients did not have interpretable post-Merci perfusion images to determine whether there was persistent ischemic penumbra following thrombectomy. Three patients in each group had either contrast staining, early petechial HT, or both on postintervention CT imaging but did not undergo sufficient imaging to determine the exact nature and were thus excluded from secondary HT analyses. The age distribution of the study cohort is displayed by decile in Table 1 .

Baseline demographics and aspects of the intervention were similar between groups (Table 2). More elderly patients had a cardioembolic source of their stroke (81\% versus 55\%, $P=.02$ ). The distribution of anterior circulation strokes was similar between groups, as was the proportion affecting the dominant hemisphere.

The rates of good recanalization, time to the first Merci pass, and the use of adjuvant therapies and concomitant intravenous or IA rtPA were similar between groups. In the younger patients, 2 underwent adjuvant PTA in the involved intracranial artery, 1 received IA abciximab, and 2 had tandem cervical ICA lesions, which were treated with carotid angioplasty and stenting, while 2 older patients underwent adjuvant intracranial PTA. Postintervention diffusion-perfusion mismatch was distributed evenly between both age groups.

Although pre-Merci NIHSS scores were similar between groups, older patients fared worse than the younger cohort on multiple clinical outcomes at discharge, including discharge NIHSS score (median, 42 versus $6.5 ; P=.006$ ), mRS score (median, 5 versus $4 ; P=.006$ ), and NIHSS improvement by 


\begin{tabular}{|c|c|c|c|}
\hline & $\begin{array}{c}\text { Age }<80 \mathrm{yr} \\
(n=75)\end{array}$ & $\begin{aligned} \text { Age } & \geq 80 \mathrm{yr} \\
(n & =31)\end{aligned}$ & \\
\hline$\overline{\text { Age }(y r)}$ & $57.5 \pm 16.0$ & $85.9 \pm 3.8$ & \\
\hline Male sex & $38(51)$ & $10(32)$ & .1 \\
\hline Dominant hemisphere or vertebrobasilar & $39(52)$ & $20(65)$ & .2 \\
\hline Anterior circulation & $70(93)$ & $30(97)$ & .7 \\
\hline \multicolumn{4}{|l|}{ Premorbid history of } \\
\hline Cardiac disease & $38(51)$ & $21(68)$ & .1 \\
\hline Hypertension & $41(55)$ & $21(68)$ & .2 \\
\hline Hyperlipidemia & $22(29)$ & $10(32)$ & .8 \\
\hline Diabetes & $16(21)$ & $2(6)$ & .9 \\
\hline Prior stroke or TIA & $3(4)$ & $1(3)$ & 1 \\
\hline Peripheral vascular disease & $4(5)$ & $2(6)$ & 1 \\
\hline Cardioembolic source & $41(55)$ & $25(81)$ & .02 \\
\hline AOL 2-3 & $58(77)$ & $25(81)$ & 8 \\
\hline Time to intervention (hr) & $6: 25 \pm 2: 49$ & $6: 21 \pm 2: 17$ & .9 \\
\hline Persistent mismatch following Merci ${ }^{\mathrm{b}}$ & $35 / 67(52)$ & $16 / 29(55)$ & .8 \\
\hline Intravenous or IA thrombolytic & $27(36)$ & $11(35)$ & 1 \\
\hline Adjunctive therapy & $6(8)$ & $2(6)$ & 1 \\
\hline Admission glucose (mg/dL) & $142 \pm 65$ & $141 \pm 42$ & .9 \\
\hline
\end{tabular}

a Baseline characteristic, No. (\%) unless otherwise specified.

b Eight patients younger than 80 year of age and 2 older did not have interpretable perfusion images.

Table 3: Clinical outcomes, secondary end points, and HT in patients undergoing Merci thrombectomy for acute ischemic stroke ${ }^{a}$

\begin{tabular}{|c|c|c|c|c|c|}
\hline & $\begin{array}{c}\text { Age }<80 y r \\
(n=75)\end{array}$ & $\begin{aligned} \text { Age } & \geq 80 \mathrm{yr} \\
(n & =31)\end{aligned}$ & $P^{b}$ & $\begin{array}{l}\text { Age } 65-79 \text { yr } \\
(n=33)\end{array}$ & $P^{b}$ \\
\hline Admission NIHSS & $18(13-22)$ & $20(18-23)$ & .1 & $17(15-23)$ & .3 \\
\hline Hospital length of stay (days) ${ }^{C}$ & $10.4 \pm 8.0$ & $8.3 \pm 5.0$ & .3 & $6.2 \pm 3.0$ & .1 \\
\hline Stroke-related death & $11(15)$ & $15(48)$ & $5.5(2.1-14.1)$ & $8(24)$ & $2.9(1.0-8.5)$ \\
\hline Discharge NIHSS & $6.5(2-42)$ & $42(6-42)$ & .006 & $11(3-42)$ & .09 \\
\hline Discharge NIHSS in survivors ${ }^{d}$ & $5(2-12)$ & $5.5(2-11)$ & 1 & $4(0-11)$ & .8 \\
\hline NIHSS change & $4.2 \pm 14.4$ & $-4.9 \pm 16.9$ & .01 & $1.7 \pm 15.8$ & .1 \\
\hline NIHSS change in survivors ${ }^{d}$ & $9.4 \pm 8.6$ & $10.4 \pm 5.5$ & .7 & $10.1 \pm 7.5$ & .9 \\
\hline Discharge mRS & $4(1-5)$ & $5(3.5-6)$ & .006 & $3(0.5-4)$ & .1 \\
\hline Discharge mRS in survivors ${ }^{d}$ & $3(1-4)$ & $3.5(2-4)$ & .9 & $2(0-4)$ & .7 \\
\hline $\begin{array}{l}m R S \leq 2 \text { in survivors } \\
H^{\mathrm{e}}\end{array}$ & $25 / 62(40)$ & 6/16 (38) & $.9(.3-2.8)$ & $11 / 23(48)$ & $0.7(.2-2.4)$ \\
\hline Any HT within $72 \mathrm{hr}$ & $30 / 72(42)$ & $17 / 28(61)$ & $2.2(.9-5.3)$ & $13 / 30(43)$ & $2.0(.7-5.8)$ \\
\hline Symptomatic HT within 72 hr & $7 / 72(10)$ & $3 / 28(11)$ & $.9(.2-3.7)$ & $2 / 30(7)$ & $1.7(.3-10.9)$ \\
\hline Parenchymal HT within $72 \mathrm{hr}$ & $9 / 72(13)$ & $4 / 28(14)$ & $1.2(.3-4.1)$ & $3 / 30(10)$ & $1.5(.3-7.4)$ \\
\hline
\end{tabular}

${ }^{a}$ Clinical measure, No. (\%) unless otherwise specified; all NIHSS and mRS values except NIHSS change are shown as median (interquartile range).

b ORs $(95 \% \mathrm{CI})$ are shown for selected comparisons.

${ }^{c}$ One patient with a prolonged hospitalization due to surgery unrelated to the stroke was excluded from LOS analysis.

d Patients who died were not analyzed in these comparisons.

e Three patients in each group did not undergo imaging by 72 hours to determine HT.

discharge $(-4.9[$ decline] versus $4.2, P=.01)$. Stroke-related deaths occurred more frequently in the elderly cohort $(48 \%$ versus $15 \%, P=.0002$ ). Table 3 displays clinical outcomes of patients by age.

In the secondary analysis, with deaths excluded, all comparisons were similar among the survivors of both groups (Table 3). The distribution of good outcomes at discharge (mRS score, $\leq 2$ ) was even among survivors of both ages (38\% versus $40 \%$; OR, 0.9; 95\% CI, 0.3-2.8). Figure 1 displays the distribution of mRS scores sorted by the 2 age groupings. Rates of varying types of HT and the hospital LOS did not vary by age.

Table 3 also shows the results of the post hoc comparison of the subgroup 65-79 years of age. The rate of stroke-related death remained higher in the patients 80 years of age and older compared with this post hoc subgroup (48\% versus 24\%; OR, 2.9; 95\% CI, 1.0-8.5). Similar to the primary analysis, the clinical outcomes in survivors were also equivalent (discharge
NIHSS score, $P=.8$; discharge $\mathrm{mRS}$ score $\leq 2,38 \%$ versus 48\%; OR, 0.7; 95\% CI, 0.2-2.4).

An unexpected baseline difference in the 2 cohorts was the uneven distribution of cardioembolic stroke source, which we further investigated post hoc. After pooling both cohorts and separating them according to whether the stroke source was cardioembolic in origin, we detected no differences in the proportion of stroke-related deaths (27\% [18/67] versus $22 \%$ [8/37]; OR, 1.3; 95\% CI, 0.5-3.4) and good clinical outcome at discharge in survivors (30\% [20/67] versus 30\% [11/37]; OR, 1.0 ; 95\% CI, 0.4-2.4).

\section{Discussion}

Recent effort to optimize patient selection for Merci thrombectomy is beginning to discriminate factors affecting outcome. Our series demonstrates that advanced age predicts overall poor outcome following Merci thrombectomy. This 


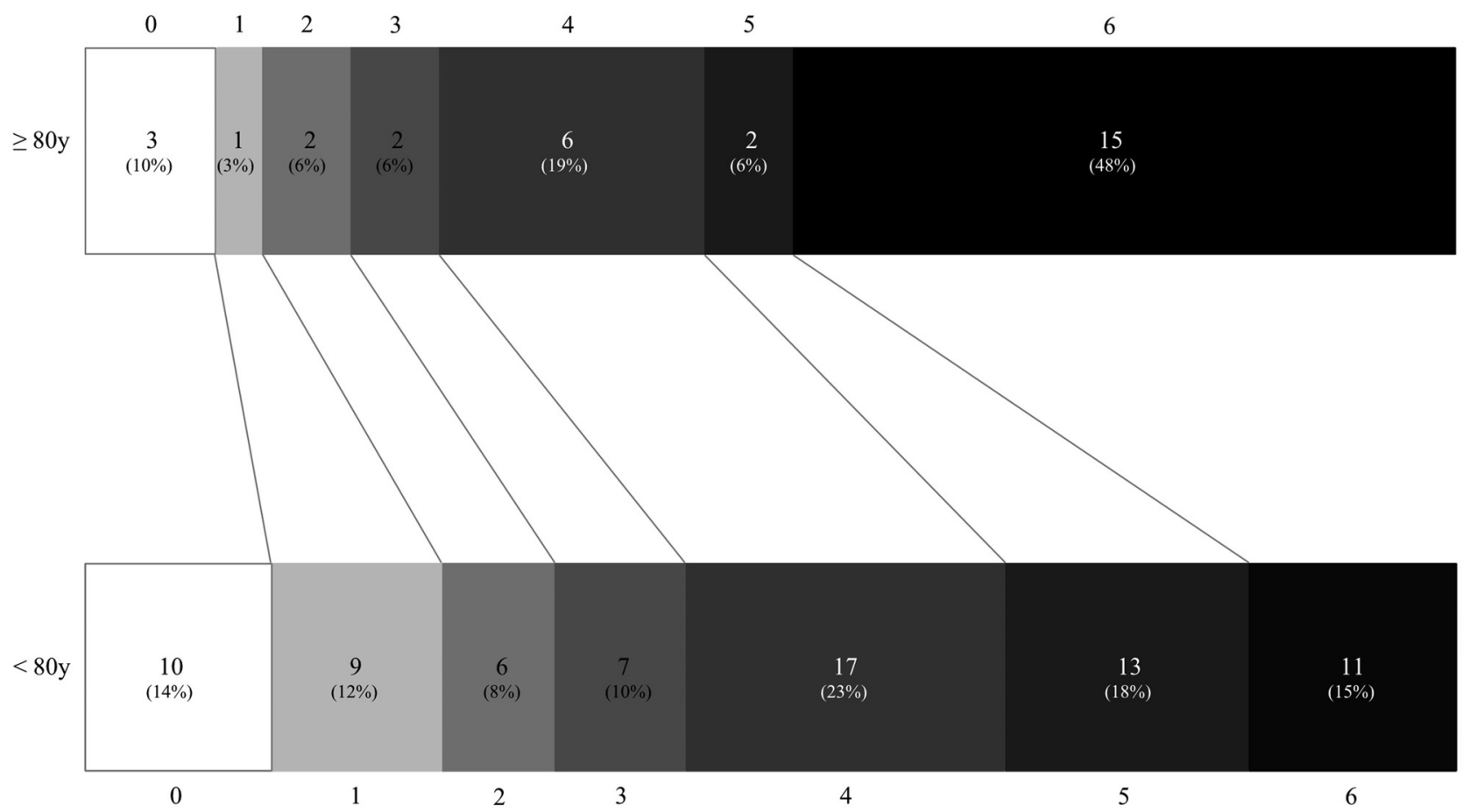

Fig 1. Discharge modified mRS scores in patients $\geq 80$ years of age versus younger patients.

disadvantage is nearly entirely due to the higher rate of strokerelated death, which occurred in nearly half of all patients 80 years of age or older. Nearly all of the clinical variables used in our study incorporate death into their measurement, namely discharge NIHSS score, change in NIHSS score, and mRS score.

Most interesting, if a patient survived the stroke, the probability of good outcome by discharge did not vary according to age. By secondarily analyzing clinical outcomes in such a dichotomized fashion, we were able to account for the impact made by family influence on decisions to withdraw care due to poor prognosis. These family variations confound all analyses incorporating mortality.

Contrary to our initial expectations, the burden of decreased neuronal plasticity accompanying advanced age does not substantially impede neurologic recovery in survivors. This may be more dependent on the fact that, in select patients with salvageable tissue, the success of recanalization has a more significant impact on outcome than age. In fact, all elderly survivors with good clinical outcomes at discharge were successfully recanalized (6/6) compared with $76 \%$ of the younger cohort $(19 / 25, P=.3)$.

By analyzing the stroke survivors separately, however, we significantly limited further comparison in our study by reducing the sample size. Because half of the elderly group was not further analyzed in a number of comparisons, our ability to detect intergroup differences was substantially limited in these instances. However, with nearly similar rates of good outcome among survivors, it appears that functional recovery is similar among differing age groups, even though the good outcome subgroup is composed solely of 6 elderly survivors. Larger samples are clearly needed to substantiate this finding.

These observations are somewhat comparable with findings in the similarly aged cohort receiving IA thrombolysis for acute ischemic stroke. ${ }^{7}$ Both groups in our study had significantly higher mortality rates by discharge ( $48 \%$ and $15 \%)$ than their counterparts in the IA rtPA group (18\% and 10\%, respectively, reported at 7 days). This finding is likely due to the presumably higher proportion of proximal occlusions in our Merci group. Alternatively, there may be some outcome effect from the greater delay in time from symptom onset to intervention in our study, 383 minutes compared with the 259 minutes average delay in our previous IA rtPA report. Similar to the current study, however, previous IA rtPA data showed that age as a dichotomous variable was not a predictor of good ( $\mathrm{mRS}$ score, $\leq 2$ ) outcome, though surviving patients were not exclusively compared in a separate analysis and disability at 90 days was the outcome measure used in the IA rtPA cohort. Preliminary analysis of the MERCI and multi MERCI cohort stratified by age has yielded similar results. ${ }^{11}$

Although IA thrombolysis is a markedly different mechanism of recanalization from mechanical thrombectomy, we found a similar recanalization rate between age groups, which is consistent with previous reports of IA thrombolytic therapy. ${ }^{6,7,12}$ Also similar to previous analyses of patients receiving IA rtPA, the rate of HT was no different between age groups, $5,6,13,14$ despite the fact that increased age is an independent risk factor for HT following intravenous thrombolysis. ${ }^{15,16}$ As with patients receiving IA thombolytics, this finding likely stems from a more rigorous patient selection process and the systemic absence of thrombolytic agents in most cases.

Although no longer significant when including stroke-related deaths, the differences in clinical outcome between the 65- and 79-year-old post hoc subgroup and those 80 years of age or older still trended in the same direction as the primary group comparisons of NIHSS score change, discharge NIHSS score, and discharge mRS score. Although this likely reflects the decrease in the size of the younger cohort from 75 to 33, the 
clinical outcomes in patients younger than 65 years of age may be worse than those the 65-79 years of age. For instance, younger patients are very likely treated aggressively despite large preintervention core infarct volumes and are less likely to be treated palliatively following failed intervention.

A limitation of this study is that it is a retrospective singlecenter observation. In addition, there were several radiographic variables that have established relationships with functional outcome that were not considered in our study, such as the angiographic collateral grade $e^{17,18}$ or the final infarct core volume. ${ }^{19,20}$ We admittedly report the AOL score, representing the final recanalization, which does not entirely reflect reperfusion. We chose the AOL scale because its definition is more constant across studies and is more applicable to the cerebral vasculature than the Thrombolysis in Myocardial Ischemia Scale, which has led to contradictory applications in past clinical trials. ${ }^{21,22}$ Several other clinical aspects such as systolic blood pressure and temperature during the hospitalization impact overall outcome ${ }^{23-25}$ though they were beyond the scope of our study. Finally, because this study only compared the immediate neurologic outcomes and disability on discharge, long-term applicability is yet to be determined.

Although we have shown that older patients are more likely to die following Merci thrombectomy regardless of recanalization success, this treatment option should not be denied purely on the basis of age considerations. Approximately $38 \%$ of elderly survivors (6/16) or nearly $20 \%$ of the total number of the elderly cohort (6/31) had good functional outcomes by discharge. In our opinion, this measures well against the invariably fatal or severely disabled outcome anticipated in comparably aged patients with untreated large-vessel occlusions.

Until a randomized trial can establish whether age should be a discriminating factor for selection, thrombectomy should remain an alternative for the elderly patient, with careful consideration of all aspects of the patient's condition. Our case series suggests, though, that older age as a variable should help guide family discussions concerning patient prognosis and should be a consideration in the design of any future trial of thrombectomy for acute stroke.

\section{Conclusions}

Increasing age predicts worse outcome, though functional recovery in survivors matches that of younger patients. Future clinical studies of acute mechanical thrombectomy should account for such age-related differences.

\section{References}

1. Gobin YP, Starkman S, Duckwiler GR, et al. MERCI 1: a phase 1 study of Mechanical Embolus Removal in Cerebral Ischemia. Stroke 2004;35:2848-54

2. Smith WS, Sung G, Starkman S, et al, for the MERCI Trial Investigators. Safety and efficacy of mechanical embolectomy in acute ischemic stroke: results of the MERCI trial. Stroke 2005;36:1432-38

3. Day J. Population Projections of the United States by Age, Sex, Race, and Hispanic Origin: 1995-2050. Washington, DC: US Bureau of the Census; 1996. Current Population Reports, Series P25-1130
4. Brown RD, Whisnant JP, Sicks JD, et al. Stroke incidence, prevalence, and survival: secular trends in Rochester, Minnesota, through 1989. Stroke 1996;27:373-80

5. Sharma JC, Fletcher S, Vassallo M. Strokes in the elderly: higher acute and 3 month mortality-an explanation. Cerebrovasc Dis 1999;9:2-9

6. Arnold M, Schroth G, Nedeltchev K, et al. Intra-arterial thrombolysis in $\mathbf{1 0 0}$ patients with acute stroke due to middle cerebral artery occlusion. Stroke 2002;33:1828-33

7. Kim D, Ford GA, Kidwell CS, et al. Intra-arterial thrombolysis for acute stroke in patients $\mathbf{8 0}$ and older: a comparison of results in patients younger than $\mathbf{8 0}$ years. AJNR Am J Neurorad 2007;28:159-63

8. Tanne D, Gorman M, Bates V, et al. Intravenous tissue plasminogen activator for acute ischemic stroke in patients aged 80 years and older: the TPA stroke survey experience. Stroke 2000;31:370-75

9. Khatri P, Neff J, Broderick JP, et al, for the IMS-I Investigators. Revascularization end points in stroke interventional trials: recanalization versus reperfusion in IMS-I. Stroke 2005;36:2400-03

10. Hacke W, Kaste M, Fieschi C, et al, for the ECASS Study Group. Intravenous thrombolysis with recombinant tissue plasminogen activator for acute hemispheric stroke: The European Cooperative Acute Stroke Study (ECASS). JAMA 1995;274:1017-25

11. Kim D, Jahan R, Saver JL, et al, for the MERCI and Multi MERCI Investigators. Mechanical thrombectomy with the Merci retriever for acute stroke in very elderly patients. In: Proceedings of the Fourth Annual Meeting of the American Society of Interventional and Therapeutic Neuroradiology, Dana Point, California, August 1, 2007

12. Furlan A, Higashida R, Weschler L, et al. Intra-arterial prourokinase for acute ischemic stroke: The PROACT II study: a randomized controlled trial. Prolyse in Acute Cerebral Thromboembolism. JAMA 1999;282:2003-11

13. Kidwell CS, Saver JL, Carneado J, et al. Predictors of hemorrhagic transformation in patients receiving intra-arterial thrombolysis. Stroke 2002;33:717-24

14. Kase CS, Furlan AJ, Wechsler LR, et al. Cerebral hemorrhage after intra-arterial thrombolysis for ischemic stroke: the PROACT II trial. Neurology 2001;57:1603-10

15. Tanne D, Kasner SE, Demchuk AM, et al. Markers of increased risk of intracerebral hemorrhage after intravenous recombinant tissue plasminogen activator therapy for acute ischemic stroke in clinical practice: the Multicenter rt-PA Stroke Survey. Circulation 2002;105:1679-85

16. Larrue V, von Kummer RR, Müller A, et al. Risk factors for severe hemorrhagic transformation in ischemic stroke patients treated with recombinant tissue plasminogen activator: a secondary analysis of the European-Australasian Acute Stroke Study (ECASS II). Stroke 2001;32:438-41

17. Bang OY, Saver JL, Buck BH, et al, for the UCLA Collateral Investigators. Impact of collateral flow on tissue fate in acute ischaemic stroke. J Neurol Neurosurg Psychiatry 2008;79:625-29. Epub 2007 Dec 12

18. Christoforidis GA, Karakasis C, Mohammad Y, et al. Predictors of hemorrhage following intra-arterial thrombolysis for acute ischemic stroke: the role of pial collateral formation. AJNR Am J Neuroradiol 2009;30:165-70

19. Nedelmann M, Wilhelm-Schwenkmezger T, Alessandri B, et al. Cerebral embolic ischemia in rats: correlation of stroke severity and functional deficit as important outcome parameter. Brain Res 2007;1130:188-96. Epub 2006 Dec 8

20. Nighoghossian N, Hermier M, Adeleine P, et al. Baseline magnetic resonance imaging parameters and stroke outcome in patients treated by intravenous tissue plasminogen activator. Stroke 2003;34:458-63

21. Tomsick T. TIMI, TIBI, TICI: I came, I saw, I got confused. AJNR Am J Neuroradiol 2007;28:382-84

22. Higashida R, Furlan A, Roberts H, et al, for the Technology Assessment Committees of the American Society of Interventional and Therapeutic Neuroradiology and the Society of Interventional Radiology. Trial design and reporting standards for intraarterial cerebral thrombolysis for acute ischemic stroke. $J$ Vasc Interv Radiol 2003;14:945-46

23. Tejima E, Katayama Y, Suzuki Y, et al. Hemorrhagic transformation after fibrinolysis with tissue plasminogen activator: evaluation of role of hypertension with rat thromboembolic stroke model. Stroke 2001;32:1336-40

24. Elewa HF, Kozak A, Johnson MH, et al. Blood pressure lowering after experimental cerebral ischemia provides neurovascular protection. $J$ Hypertens 2007;25:855-59

25. Greer DM, Funk SE, Reaven NL, et al. Impact of fever on outcome in patients with stroke and neurologic injury: a comprehensive meta-analysis. Stroke 2008;39:3029-35 